\title{
ESTUDO EXPLORATÓRIO SOBRE A DISTRIBUIÇÃO DE USUÁRIOS DA REDE MUNICIPAL ESPECIALIZADA EM DST/AIDS EM RELAÇÃO À DISTÂNCIA DE SUAS RESIDÊNCIAS AOS SERVIÇOS. MunicíPIO de SÃo PAULO, 2009
}

\author{
Katia Cristina Bassichetto ${ }^{1}$ \\ Maria Cristina Haddad Martins ${ }^{2}$
}

\begin{abstract}
Resumo: Estudos sobre a distância entre residência dos usuários e serviços de saúde orientam políticas de referência e contra referência, podendo vir a minimizar grandes deslocamentos e facilitar a expansão do acesso aos serviços. Neste estudo, utilizaram-se dados de uma pesquisa sobre Avaliação do Perfil Nutricional de Pessoas Vivendo com HIV e Aids (PVHA) da Rede Municipal Especializada em DST/aids, no Município de São Paulo para descrever a distribuição dos usuários. Esta população foi mapeada por endereço de residência, assim como os serviços, onde é atendida. Construíram-se bandas de cinco em cinco $\mathrm{km}$ em torno dos serviços e calculou-se a frequência de residentes em cada banda. Considerou-se "alta concentração" quando mais de 80\% dos usuários residem na faixa de 10 $\mathrm{km}$. Foi possível conhecer a frequência dos usuários que residem fora do município (8,9\%), a qual variou de 2,4 a 26,7\% por serviço emapear 95,7\% dos residentesno MSP. Em 10 dos 12 serviços há alta concentração de residentes num raio de $10 \mathrm{~km}$, variando de 81,9 a 98,8\%. Esta significativa concentração de usuários residindo próximo aos serviçosreflete certa racionalidade administrativa, o que pode contribuir para a adesão ao tratamento, evidenciando os que necessitam de maior atenção.
\end{abstract}

Palavras-chave: Serviços de saúde; HIV/AIDS; Acessibilidade geográfica.

\section{EXPLORATORY STUDY ON THE DISTRIBUTION OF MUNICIPAL SPECIALIZED DST/AIDS NETWORK'S USERS ACCORDING TO THE DISTANCE FROM THEIR HOMES TO THE SERVICES. MUNICIPALITY OF SÃO PAULO, 2009}

\begin{abstract}
Studies on the distance between residence and place of attendance of users of secondary health care services orientate reference policies and against-reference, minimize large displacements and facilitate the expansion of access. In this sense, it was used data from a survey on evaluation of the Nutritional Profile of people living with HIV and Aids (PLWHA), Network Services in Specialized Municipal in DST/aids(RME), in the municipality of São Paulo (MSP) to describe the distribution of PLWHA, users of this network. This population was mapped by the address of residence, as well as the 12 services of RME in DST/aids, where this population is attend. Were built bands of five $\mathrm{km}$ in five $\mathrm{km}$ around services and calculated the frequency of users residing within each band. It was considered "high concentration" when more than 80 percent of users reside within the range of $10 \mathrm{~km}$.It was possible to know the frequency of users residing outside the municipality (8.9\%), which

\footnotetext{
${ }^{1}$ Graduação em Nutrição pelo Centro Universitário São Camilo, mestrado em Epidemiologia pela Universidade Federal de São Paulo, doutorado em Infectologia em Saúde Pública - Coordenadoria de Controle de Doenças - Secretaria de Estado da Saúde. Assessora técnica da Coordenação de Epidemiologia e Informação da Secretaria de Saúde da Prefeitura do Município de São Paulo. E-mail: kcbassi@gmail.com

${ }^{2}$ Graduação em Arquitetura e Urbanismo pela Universidade de São Paulo, doutorado em Ciências pela Faculdade de Medicina da Universidade de São Paulo. Gerente da Gerência de Geoprocessamento e Informações Sociambientais/GISA da Coordenação de Epidemiologia e Informação/CEInfo da Secretaria Muncipal de Saúde de São Paulo. E-mail: mcrish@gmail.com DOI: 10.7154/RDG.2012.0024.0007
} 
ranged from 2.4 to 26.7 percent per service and to map 95.7 percent of users that reside in the MSP. It was noted that in 10 of 12 services there is high concentration of users residing within a radius of $10 \mathrm{~km}$, which varied from 81.9 to 98.8 percent. This significant concentration of users residing next to services reflects certain administrative rationality, which may contribute to treatment adherence, highlighting those who require greater attention.

Key-Words: health services; HIVaids; geographical accessibility.

\section{INTRODUÇÃO}

Na história da epidemia da aids (Síndrome da Imunodeficiência Adquirida) na cidade de São Paulo foram notificados, entre 1980 e dezembro de 2008, um total 71.508 casos, sendo 69.274 adultos, 2.126 crianças e 108 casos de idade ignorada, o que corresponde a 15,7\% dos casos de aids do país (CIDADE DE SÃO PAULO, 2009); (BRASIL, 2009). A atenção às pessoas vivendo com HIV e aids segue os princípios e diretrizes do Sistema Único de Saúde (SUS), destacando-se a universalidade de acesso aos serviços de saúde, em todos os níveis de assistência; a integralidade de assistência, entendida como conjunto articulado e contínuo das ações e serviços preventivos e curativos, individuais e coletivos, exigidos para cada caso em todos os níveis de complexidade do sistema e a descentralização políticoadministrativa, com direção única em cada esfera de governo, com ênfase na regionalização e hierarquização da rede de serviços de saúde, em níveis de complexidade crescente. Estes princípios constam da Constituição de 1988 e da Lei № 8080/90, que regula, em todo o território nacional, as ações e serviços de saúde, dispondo sobre as condições para a promoção, proteção e recuperação da saúde, a organização e o funcionamento dos serviços correspondentes (BRASIL, 1988); (BRASIL, 1990).

Especificamente, em relação à regionalização, mais recentemente, o Pacto pela Saúde adota uma série de estratégias voltadas à organização da rede pública, visando sua integração e resolutividade, garantia de acesso, qualidade às ações e dos serviços de saúde; a racionalização dos gastos e a otimização da aplicação dos recursos. Adquire um caráter inovador, baseando-se em um modelo de gestão compartilhada entre as três esferas do governo. Para preservar a interdependência entre estes níveis de gestão, fundamentais para a redução das desigualdades territoriais, foram definidos os seguintes pressupostos: territorialização, flexibilidade, cooperação, cogestão, financiamento solidário, participação e 
controle social, possibilitando ganhos de escala nas ações e serviços de abrangência regional (BRASIL, 2007).

Estes pressupostos guardam relação com o desenvolvimento das teorias a respeito do conceito de região, que tem incorporado a complexidade contemporânea com novos vetores de modernização e novas possibilidades de usos do território. Dessa forma, a concepção e a condução de políticas públicas necessitam incorporar um conceito dinâmico de espaço geográfico e enfocar a questão regional como principal estratégia para enfrentamento das desigualdades sociais. O conceito de "região de saúde", em particular, considera a base territorial de planejamento e organização da atenção à saúde de acordo com as características demográficas, socioeconômicas, geográficas, sanitárias, epidemiológicas e de oferta de serviços, entre outras (ALBUQUERQUE; IOZZI, 2011). Na cidade de São Paulo, a organização de serviços para a atenção de pessoas vivendo com HIV e aids acompanhou a dinâmica da epidemia ao longo dos anos, evoluindo de apenas dois serviços especializados em 1991 - ano de implantação do Programa Municipal de DST/AIDS para 15 serviços em 1996. Atualmente, a Rede Municipal Especializada em DST/aids (RME) conta com 24 serviços, que recebem denominação específica segundo sua complexidade: Centros de Testagem e Aconselhamento (CTA), Ambulatórios de Especialidades (AE), Serviços de Assistência Especializada (SAE) e Centros de Referência (CR). As pessoas diagnosticadas com DST/HIV/aids podem receber atendimento em quaisquer destes serviços, com exceção dos CTA, o que totaliza 15 serviços de assistência distribuídos em diversas regiões da cidade (Figura 1). Os testes sorológicos, que permitem o diagnóstico da infecção pelo HIV estão disponíveis atualmente em serviços de vários níveis de atenção da Rede Municipal de Saúde da cidade de São Paulo.

No entanto, preconiza-se que as pessoas com diagnóstico positivo para o HIV, sejam referenciadas para serviços de atenção secundária, onde se encontram, por exemplo, profissionais especializados e realizam-se procedimentos de alto custo, como exames para monitoramento da evolução da doença e disponibilização de tratamento com antirretrovirais, quando necessário. Em dezembro de 2008, encontravam-se em seguimento, nesta rede especializada, cerca de 20.450 pessoas vivendo com HIV e aids (Cidade de São Paulo, 2009), contingente significativamente superior ao atendido por outros serviços disponíveis na cidade. 
O acesso da população aos serviços de saúde é um pré-requisito de fundamental importância para uma eficiente assistência à saúde. A localização geográfica dos serviços é um dos fatores que interferem nesta acessibilidade (UNGLERT et al, 1987); (UNGLERT, 1990). A RME da cidade de São Paulo levou em conta, para sua implantação, a magnitude da doença e a distribuição dos casos no território. Foram considerados os conceitos de áreas de abrangência e de influência de forma a implementar algum grau de racionalidade administrativa para atendimento dos potenciais usuários destes serviços.

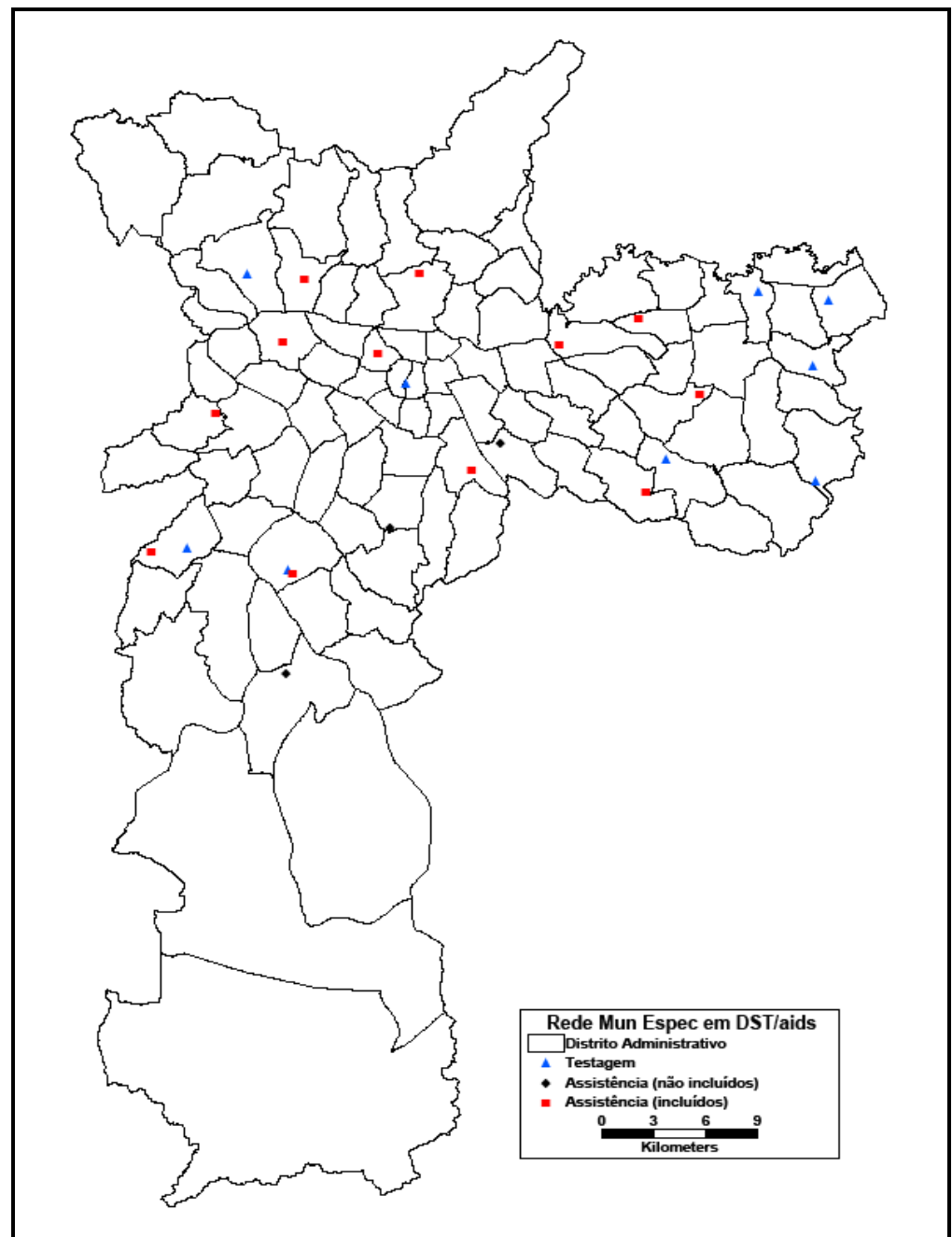

Figura 1 - Rede Municipal Especializada em DST/aids de São Paulo, por tipo de serviços, incluídos e não incluídos no estudo, 2009.

A área de influência de um serviço de saúde refere-se à procedência dos usuários que efetivamente buscam por suas ações de saúde e reflete a pressão da demanda espontânea da população por atenção, onde nem sempre o local mais próximo, geograficamente é o mais apropriado. Já a área de abrangência é representada pela área geográfica definida como de responsabilidade da equipe de saúde local, a partir de critérios de acessibilidade e 
fluxos da população. Cabe à unidade de saúde conhecer a situação de saúde da população dessa área e se responsabilizar por ela, mesmo que não desenvolva todas as ações de saúde para aquela população (TEIXEIRA; MELO, 1995).

A acessibilidade aos serviços de saúde pode ser analisada de acordo com distintas abordagens: geográfica, representando não só à distância a ser percorrida como também as barreiras geográficas a serem transpostas; funcional (horário de funcionamento, qualidade da atenção, disponibilidade de instalações e de profissionais, tipos de programas e ações de saúde oferecidos); cultural (inserção dos serviços nos hábitos e costumes da população) e econômica (possibilidade da atual oferta de serviços não estar, na sua totalidade, desvinculada de algum sistema de pagamento por parte do usuário) (TEIXEIRA; MELO, 1995). Alguns autores acrescentam a questão da aceitabilidade dos usuários, como elemento de análise. Em um modelo racionalizador desenvolvido por Donabedian a acessibilidade é apresentada como "um fator mediador entre a capacidade de produzir serviços e a produção ou consumo real dos ditos serviços", apresentando a seguinte definição: "algo adicional à mera presença ou disponibilidade de um recurso em certo lugar e em um dado momento. Compreende as características do recurso que facilitam ou dificultam o uso por parte dos potenciais clientes"(DONABEDIAN, 1988).

Outros autores combinam os atributos dos recursos e os da população em seus conceitos de acessibilidade, supondo-se que exista uma série de características que se justapõem e que estão associadas à questão do acesso, como, por exemplo, a acessibilidade financeira, definida como capacidade individual para custear a atenção médica e a acessibilidade física, como meios de transporte, tempo e procura de informações adicionais, que supõem o processo de procura da atenção (FRENK, 1985). Esta diversidade de sentidos pode trazer alguma dificuldade para a compreensão do referencial adotado em situações específicas. Observa-se que os estudos sobre acesso de pessoas vivendo com HIV/aids a serviços de saúde investigam principalmente os fatores associados à adesão à medicação antirretroviral, comparando países desenvolvidos com os em desenvolvimento, a influência da raça/cor, entre outros, sendo o acesso geográfico muito pouco estudado (NEMES et al, 2009); (DO LAGO; COSTA, 2009); (GIULIANO; VELLA, 2007), (COHEN, 2007).

Outro aspecto analisado pelos estudos são os padrões de distribuição espacial de casos de aids, em determinados territórios, ao longo do tempo, utilizando recursos de geoprocessamento, com incorporação de análise estatística espaço-temporal. Estes estudos 
têm permitido maior compreensão da dinâmica da epidemia, considerando os diferentes segmentos atingidos e podem vir a contribuir na determinação de modelos espaciais mais precisos de previsão da distribuição de novos casos (DIAS; NOBRE, 2001); (SZWARCWALD, 2001); (TOMAZELLI, 2003). Há estudos mais recentes, no entanto, que buscaram analisar a questão do fluxo dos serviços de assistência às pessoas vivendo com HIV e aids, considerando o processo de interiorização da epidemia, e principalmente a incidência em indivíduos residentes em municípios de pequeno porte, que inevitavelmente precisam se deslocar para obter assistência especializada. Estes estudos apontam a dimensão do "viver longe do serviço de saúde" como um dos fatores dificultadores do acesso e da continuidade do uso dos serviços, o que poderia potencialmente sinergir para aumentar a vulnerabilidade de determinados indivíduos (OLIVEIRA, 2009).

Este panorama reforça a importância de estudos sobre acessibilidade a serviços de atenção à saúde, na perspectiva geográfica, tendo em vista que podem contribuir para orientar políticas de regulação, minimizar os deslocamentos e ampliar o acesso.

Neste sentido, considerou-se oportuno utilizar dados de uma pesquisa sobre Avaliação do Perfil Nutricional de Pessoas Vivendo com HIV e Aids, coletados em 2008 e 2009, em serviços de assistência da RME, na cidade de São Paulo (CIDADE DE SÃO PAULO, 2010), para descrever a distribuição dos usuários desta rede, em relação à distância entre local de residência e serviços de assistência, bem como a proporção de população de outros municípios da Região Metropolitana de São Paulo (RMSP), atendida em cada serviço.

Este estudo, ao analisar retrospectivamente a distância da residência de usuários a serviços de atenção secundária ao SUS e, que, portanto reflete escolhas de trajetos efetivamente concretizadas, pode contribuir para ampliar a compreensão sobre as motivações envolvidas nestas escolhas, iluminando um aspecto importante da interação entre serviços e usuários e inspirar outros estudos que aprofundem a análise dos fatores que dificultam ou facilitam o acesso.

\section{MATERIAL E MÉTODO}

Dos 15 serviços de assistência da RME , 12 foram incluídos no estudo original. Estes serviços e a população de estudo, moradora no MSP, segundo endereço de residência, foram mapeados por meio da utilização do software Maptitude 4.6 (CALIPER CORPORATION). 
Previamente ao mapeamento, foi necessário padronizar endereços do banco de dados da pesquisa, para torná-los iguais aos da base geográfica. Este procedimento foi realizado com a utilização de um aplicativo desenvolvido pela Empresa de Tecnologia da Informação e Comunicação do Município de São Paulo - PRODAM, que compara o endereço do banco de dados a ser mapeado com a denominação de logradouros que se encontra na base geográfica.

Como resultado, é criado um novo campo naquele banco para inserção do endereço em formato padronizado, permitindo assim que o mapeamento por endereço seja realizado. Foram construídas bandas circulares de cinco em cinco km, em torno de cada serviço, e calculada a frequência de usuários residentes dentro de cada banda, através de recursos de geoprocessamento. Considerou-se como "alta concentração", quando mais de $80 \%$ dos usuários residiam dentro da faixa de $10 \mathrm{~km}$, tendo em vista a extensão geográfica da cidade e o fato de tratarem-se de serviços de atenção secundária. Calculou-se, também, a frequência de usuários residentes fora do município.

\section{RESULTADOS}

A população estudada é de 950 PVHA, participantes da pesquisa sobre perfil nutricional, das quais 85 (8,9\%) eram residentes em outros municípios da Região Metropolitana de São Paulo, o que já era esperado, tendo em vista a baixa disponibilização de serviços de assistência especializada em DST/aids nestes municípios (apenas seis serviços, em 38 municípios, à época da pesquisa), segundo o Cadastro Nacional de Estabelecimentos de Saúde.

Dos 865 residentes no MSP, foi possível mapear 828 (95,7\%), o que pode ser considerado uma boa proporção de mapeamento. Este resultado pode ser decorrência de se tratarem de dados originários de pesquisa, na qual os pesquisadores foram bem orientados com relação à forma de preenchimento de todos os campos do formulário incluindo os relacionados ao endereço. 
Tabela 1 - Distribuição de usuários dos serviços de assistência especializada em DST/aids, segundo distância entre o local de residência e o de atendimento. Muncípio de São Paulo, 2009.

\begin{tabular}{|c|c|c|c|c|c|c|c|c|c|c|c|c|}
\hline \multirow{3}{*}{ Unidade de Saúde } & \multicolumn{7}{|c|}{ residentes no MSP } & & & \multirow{3}{*}{$\begin{array}{c}\text { não } \\
\text { mapeados }\end{array}$} & \multirow{3}{*}{ mapeados } & \multirow{3}{*}{ total } \\
\hline & \multicolumn{2}{|c|}{$<5 \mathrm{~km}$} & \multicolumn{2}{|c|}{$<10 \mathrm{~km}$} & \multicolumn{2}{|c|}{$>10 \mathrm{~km}$} & \multirow[t]{2}{*}{ total } & outros mu & os & & & \\
\hline & $\mathbf{n}$ & $\%$ & $\mathbf{n}$ & $\%$ & $\mathrm{n}$ & $\%$ & & n & $\%$ & & & \\
\hline CR DST/aids STO AMARO & 9 & 26.5 & 29 & 85.3 & 5 & 14.7 & 34 & 2 & 5.6 & 3 & 36 & 39 \\
\hline SAE DST/aids CIDADE LIDER & 32 & 38.6 & 82 & 98.8 & 1 & 1.2 & 83 & 2 & 2.4 & 5 & 85 & 90 \\
\hline SAE DST/aids SANTANA & 25 & 42.4 & 53 & 89.8 & 6 & 10.2 & 59 & 9 & 13.2 & 7 & 68 & 75 \\
\hline SAE DST/aids FIDELIS RIBEIRO & 64 & 43.8 & 113 & 77.4 & 33 & 22.6 & 146 & 16 & 9.9 & 1 & 162 & 163 \\
\hline CRS DST/aids PENHA & 48 & 51.1 & 77 & 81.9 & 17 & 18.1 & 94 & 4 & 4.1 & 1 & 98 & 99 \\
\hline SAE DST/aids LAPA & 6 & 54.5 & 10 & 90.9 & 1 & 9.1 & 11 & 4 & 26.7 & 0 & 15 & 15 \\
\hline SAE DST/aids JD MISUTANI & 11 & 68.8 & 12 & 75.0 & 4 & 25.0 & 16 & 2 & 11.1 & 1 & 18 & 19 \\
\hline SAE DST/aids CAMPOS ELISEOS & 60 & 69.0 & 73 & 83.9 & 14 & 16.1 & 87 & 11 & 11.2 & 5 & 98 & 103 \\
\hline SAE DST/aids BUTANTÃ & 54 & 74.0 & 69 & 94.5 & 4 & 5.5 & 73 & 16 & 18.0 & 2 & 89 & 91 \\
\hline SAE DST/aids IPIRANGA & 36 & 75.0 & 44 & 91.7 & 4 & 8.3 & 48 & 6 & 11.1 & 2 & 54 & 56 \\
\hline SAE DST/aids BETINHO & 56 & 77.8 & 67 & 93.1 & 5 & 6.9 & 72 & 3 & 4.0 & 2 & 75 & 77 \\
\hline CR DST/aids FREGUESIA DO Ó & 87 & 82.9 & 99 & 94.3 & 6 & 5.7 & 105 & 10 & 8.7 & 9 & 115 & 124 \\
\hline total & 488 & 58.9 & 728 & 87.9 & 100 & 12.1 & 828 & $85^{\prime}$ & 9.3 & 38 & 913 & 951 \\
\hline
\end{tabular}

Observou-se que, em 10 dos 12 serviços participantes do estudo, há uma alta concentração de usuários residentes num raio de 10 km, que variou de 81,9 a 98,8\% (Fig. 2 a 13 e Tab. 1). Se considerarmos os usuários residentes num raio de cino $\mathrm{km}$, observou-se que seis serviços apresentaram distribuição que variou de 68,8 a $82,9 \%$, o que pode significar um resultado acima do esperado para este nível de atendimento. A proporção de residentes de fora do MSP variou de 2,4 a $26,7 \%$ por serviço.

Observou-se que os dois serviços que apresentam maior proporção de usuários advindos de outros municípios da Região Metropolitana são os SAE DST/aids Lapa e Butantã, ambos localizados na Região Oeste da cidade. Os municípios vizinhos próximos a essa Região, com exceção de Barueri, não contavam, à época deste estudo, com este tipo de serviço especializado, além de haver facilidade de acesso por transporte público, o que pode ter contribuindo para esse resultado (Fig. 6 e 12). Por outro lado, se considerarmos o número absoluto, verifica-se que o SAE DST/aids Fidélis Ribeiro, recebeu o mesmo número de usuários externos ao município que o SAE Butantã. Embora este número represente uma proporção menor, em relação ao total de usuários do serviço $(9,9 \%)$, este resultado parece indicar outro eixo de atração, também viabilizado pela facilidade de serviços públicos de transporte (Figura 10). 


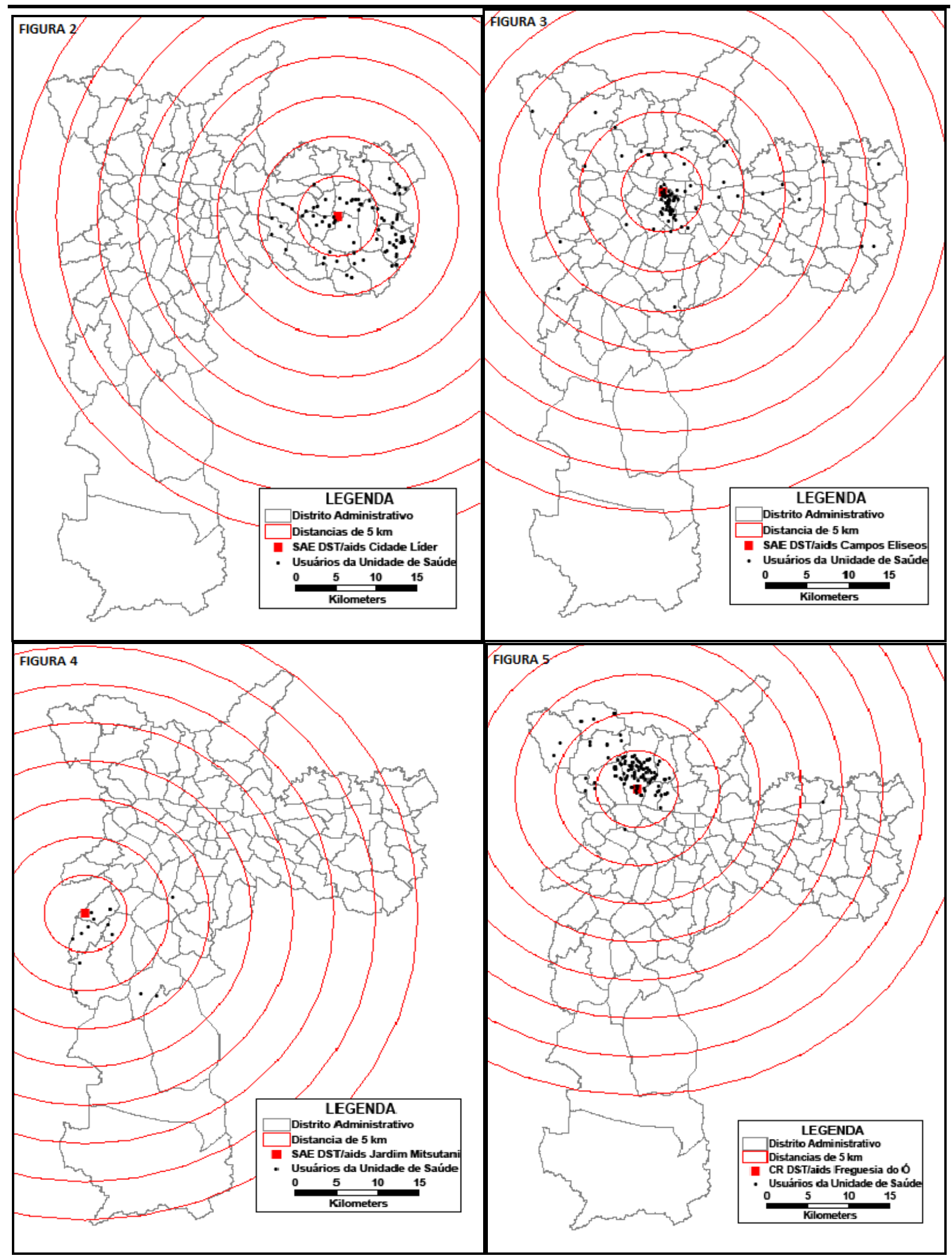




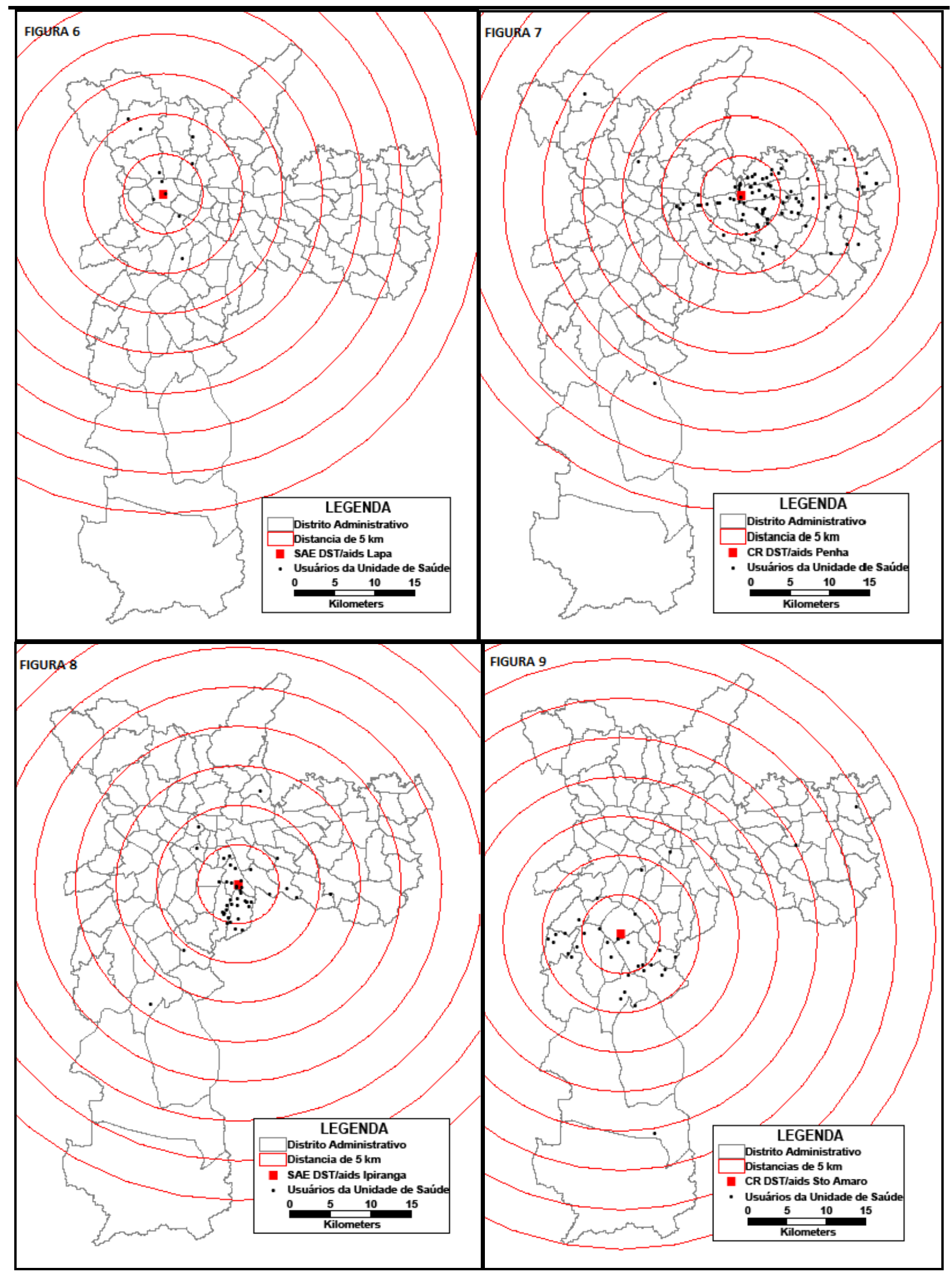




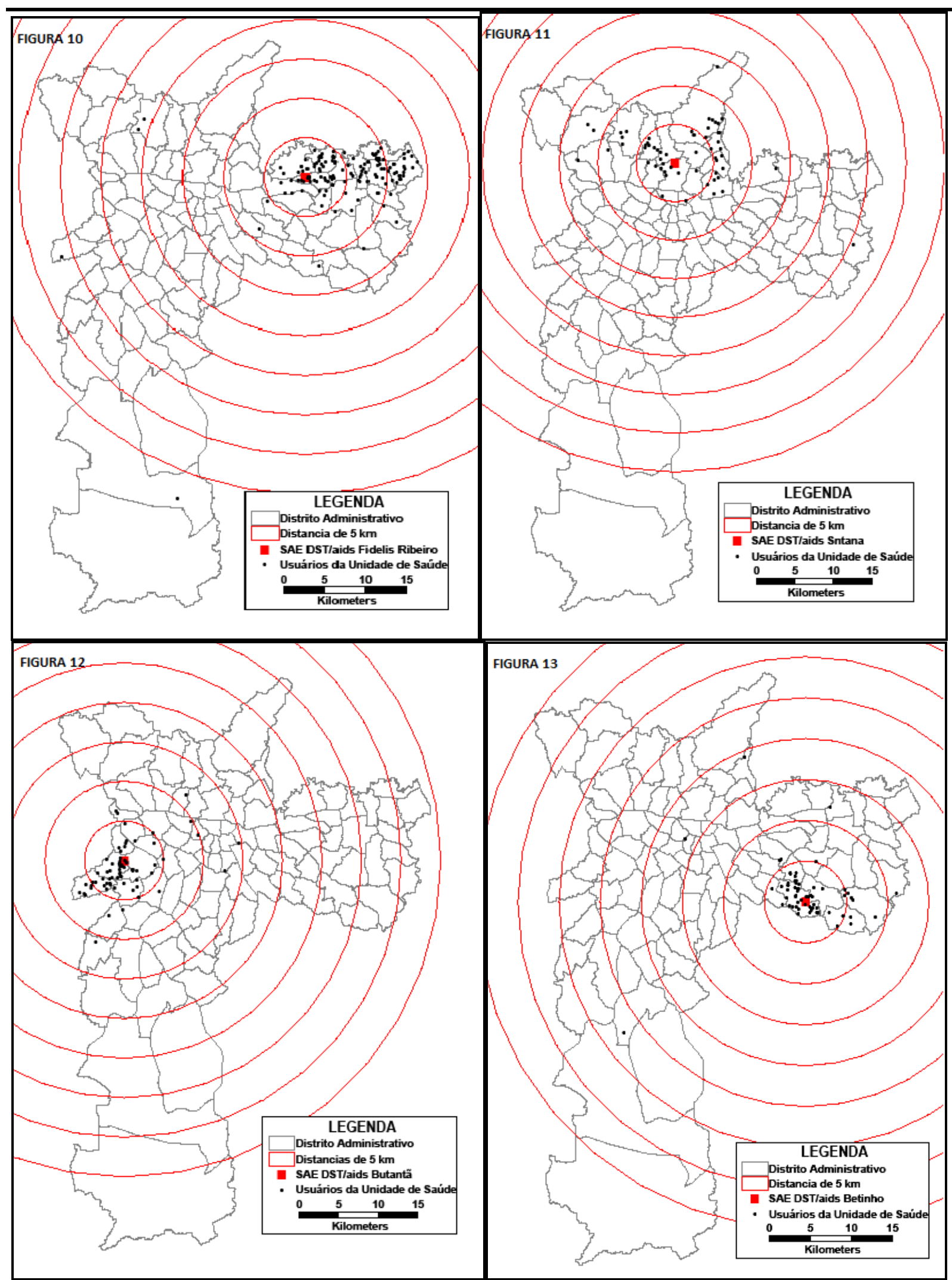

Figuras 2 a 13 - Mapeamento de usuários da Rede Municipal Especializada em DST/aids, segundo local de residência e distância dos serviços de atendimento, incluídos na pesquisa sobre Perfil Nutricional de Pessoas Vivendo com HIV/aids. Município de São Paulo, 2009. 


\section{Discussão}

A variação observada na proporção de usuários externos ao Município deve-se provavelmente a diferenças na disponibilidade de transporte público, além da pouca oferta de serviços desse tipo nos municípios vizinhos. A literatura que trata da questão do acesso a este tipo de serviço mostrou-se muito mais voltada para a utilização dos demais conceitos, que não o geográfico, conforme mencionado no presente trabalho. Há indicações, no entanto, de que a maior distância geográfica aos serviços representa um dos fatores de não adesão a protocolos terapêuticos, incluindo o tratamento antirretroviral necessário para o controle da doença e para a manutenção da qualidade de vida destes usuários (COLOMBRINI, 2009); (GIR et al, 2005); (FERRAZ; CAMPOS, 2010).

No presente estudo este aspecto não foi investigado na pesquisa original, entretanto seria recomendável, frente a estes achados, que novos estudos fossem realizados neste sentido. Entre outras questões, seria indicado avaliar se estes usuários têm utilizado eventuais estratégias, como a indicação de endereço de moradia não verídico, para obter acesso a um determinado serviço de preferência; se mudaram de endereço, para permanecer nas imediações do serviço ou optaram por serviços mais distantes da moradia, por receio do preconceito que envolve a doença.

A busca ativa de usuários faltosos aos serviços especializados em DST/aids tem sido utilizada como estratégia de rotina para minimizar os fatores que levam à não adesão. Apesar do cuidado de obtenção de consentimento para o contato com este usuário, no momento de sua matrícula, têm sido identificadas dificuldades adicionais para que esta estratégia se torne efetiva como manter de forma periódica a atualização destes contatos e ou manter sigilo sobre o tipo de serviço de saúde que está realizando a busca, dado o estigma sobre a doença ainda presente.

\section{CONCLUSÕES}

Este estudo exploratório permitiu descrever a distribuição dos usuários da RME, a partir da distância entre local de residência e serviços de assistência, bem como a proporção de população de outros municípios da Região Metropolitana de São Paulo (RMSP), atendida em cada serviço. 
Foi observado que, apesar de se tratarem de serviços públicos de atenção secundária, isto é, aqueles que disponibilizam equipe multidisciplinar especializada, há uma concentração significativa de usuários residentes próximos aos serviços analisados, podendo contribuir para a adesão ao tratamento, em se considerando a necessidade de retornos periódicos para acompanhamento.

Este resultado pode refletir, ao menos em parte, os esforços em implementar mecanismos de racionalização desta rede, sendo necessário, no entanto, dedicar maior atenção à adesão dos que não se encontram nessa condição. Os dados levantados motivam o interesse pela elucidação dos fatores que determinam a busca, o acesso e a adesão de pessoas vivendo com HIV e aids a serviços especializados e por colocar em questão os critérios atualmente utilizados para delimitação das áreas de abrangência, o que se espera possa ser objeto de novas investigações.

\section{REFERÊNCIAS BIBLIOGRÁFICAS}

ALBUQUERQUE, M.V.; IOZZI, FL. Novos rumos da regionalização da saúde: o complexo regional da saúde no estado de São Paulo/Brasil. Disponível em www. http://observasaude.fundap.sp.gov.br/observatorio/Participantes/Paginas/Default.aspx (Acessado em Outubro de 2011).

BRASIL. Constituição da República Federativa do Brasil, Brasília, DF, 1988. Disponível em: http://www.senado.gov.br/legislacao/const/con1988/CON1988_05.10.1988/CON1988.pdf

BRASIL. Lei $n=8.080$, de 19 de setembro de 1990. Dispõe sobre as condições para a promoção, proteção e recuperação da saúde, a organização e o funcionamento dos serviços correspondentes e dá outras providências. Disponível em: http://www.planalto.gov.br/ccivil_03/Leis/L8080.htm

BRASIL. Ministério da Saúde. Secretaria-Executiva. Departamento de Apoio à Descentralização. Regionalização solidária e cooperativa: orientaçõespara sua implementação no SUS / Ministério da Saúde, Secretaria-Executiva, Departamento de Apoio 
à Descentralização. - Brasília: Ministério da Saúde, 2007. 48p. (Serie A. Normas e Manuais Técnicos) (Serie Pactos pela Saúde; v. 3)

BRASIL. Ministério da Saúde, Secretaria de Vigilância em Saúde, Departamento de DST, Aids e Hepatites Virais. Boletim Epidemiológico AIDS. Brasília: Ano VI, no 01., 2009. Disponível em: http://www.aids.gov.br/sites/default/files/Boletim2010_0.pdf

CALIPER CORPORATION. MaptitudeGeographiclnformation System for Windows. Version 4.6. USA [software].

COLOMBRINI, M.R.C.; LOPES, M.H.B.M.; FIGUEIREDO, R.M. Adesão à terapia antirretroviral para HIV/Aids. Rev. Esc. Enf.USP.V.40, n.4, São Paulo, 2006.

COHEN, G.M. Access to diagnostics in support of HIV/AIDS and tuberculosis treatment in developing countries. AIDS; 21 Suppl4:S81-7, 2007.

DIAS, P.R.T.P.; NOBRE, F.F. Análise dos padrões de difusão espacial dos casos de AIDS por estados brasileiros. Cad. Saúde Pública, Rio de Janeiro, 17(5):1173-1187, 2001.

DO LAGO, R.F.; COSTA, N. do R. Antiretroviral manufacturers and the challenge of universal access to drugs through the Brazilian National STD/AIDS Program. CadSaude Publica; 25(10):2273-84, 2009.

DONABEDIAN, A. Los espacios de lasalud: Aspectos fundamentales de laorganización de la atención médica. 1a ed. em español, Mexico, 1988.

FRENK, J. El concepto y lamedición de accessibilidad. Salud Publica de Mexico, 1985. FERRAZ, T.L.B. e CAMPO,S M.R. HIV/aids: evolução histórica, aspectos psicoemocionais da convivência com a doença e a participação do farmacêutico na adesão ao tratamento. Trabalho apresentado no 8a Mostra Acadêmica UNIMEP. Piracicaba, 2010.

GIR, E.; VAICHULONIS, C.G.; OLIVEIRA, M.D. Adesão à terapêutica antirretroviral por indivíduos com HIV/aids assistidos em uma instituição do interior paulista. Rev. LatinoAm.Enfermagem. V.13, n.5, Ribeirão Preto, 2005. 
GIULIANO, M.; VELLA, S. Inequalities in health: access to treatment for HIV/AIDS. Ann IstSuper Sanita; 43(4):313-6, 2007.

NEMES, M.I.; CASTANHEIRA, E.R.; HELENA, E.T.; MELCHIOR, R.; CARACIOLO, J.M.; BASSO, C.R.; ALVES, M.T.; ALENCAR, T.M.; FERRAZ, D.A. Adesão ao tratamento, acesso e qualidade da assistência em Aids no Brasil. RevAssocMed Bras;55(2):207-12, 2009 .

OLIVEIRA, I.B.N. Acesso universal? Obstáculos ao acesso, continuidade do uso e gênero em um serviço especializado em HIV/AIDS em Salvador, Bahia, Brasil. Cad. Saúde Pública, Rio de Janeiro, 25 Sup2: S259-S268, 2009.

SÃO PAULO (Cidade). Prefeitura do Município de São Paulo. Secretaria Municipal de Saúde Programa Municipal de DST/Aids. (2007) V Inventário de Pesquisas em DST/Aids. Perfil nutricional de pessoas vivendo com HIV/Aids acompanhadas na rede Municipal Especializada em DST/Aids da Cidade de São Paulo, pg. 62. São Paulo.

SÃO PAULO (cidade). Prefeitura do Município de São Paulo. Secretaria Municipal de Saúde Programa Municipal de DST/Aids. Boletim Epidemiológico de Aids do Município de São Paulo. Ano XIII. n.12, 2009.

SZWARCWALD, C.L.; BASTOS, F.I.; BARCELLOS, C.; ESTEVES, M.A.P.; CASTILHO, E.A. Dinâmica da epidemia de AIDS no Município do Rio de Janeiro, no período de 1988-1996: uma aplicação de análise estatística espaço-temporal. Cad. Saúde Pública, Rio de Janeiro, 17(5):1123-1140, 2001.

TEIXEIRA, C.F.; MELO, C. (organizadoras). Construindo Distritos Sanitários: A experiência da Cooperação Italiana no Município de São Paulo. Ed. Hucitec. Cooperação italiana em Saúde, São Paulo-Salvador, 1995.

TOMAZELLI, J.; CZERESNIA, D.; BARCELLOS, C. Distribuição dos casos de AIDS em mulheres no Rio de Janeiro, de 1982 a 1997: uma análise espacial. Cad. Saúde Pública, Rio de Janeiro, 19(4): 1049-1061, 2003.

UNGLERT, C.V.S. O enfoque da acessibilidade no planejamento da localização e dimensão de serviços de saúde. Revista de Saúde Pública, São Paulo, 24(6): 445-52, 1990. 
UNGLERT, C.V.S; ROSENBURG, C.P.; JUNQUEIRA, C.B. Acesso aos serviços de saúde: uma abordagem de geografia em saúde pública. Revista de Saúde Pública, São Paulo, 21(5): 43946, 1987.

Artigo recebido em 01/03/2012.

Artigo aceito em 12/06/2012. 